\title{
The Care Programme Approach in learning disability psychiatry
}

\author{
Ashok Roy
}

For nearly three decades, closure of large psychiatric and learning disability hospitals has continued and more people with mental health needs remain in the community. With this continuing decrease in hospital provision, there has been an increased focus on the development of systems of safe care and effective treatment for people with significant mental health problems living in the community. Attempts to make this a central plank of mental health care have been reflected in legislation and guidance produced by the Department of Health. The Care Programme Approach (CPA) was introduced in 1991 after the publication of HC (90) 23/LASSL (90) 11 and subsequent guidance from the White Paper Caring for People (Department of Health, 1990).

The CPA was to be implemented by health authorities and trusts and was to assess people with mental health needs and focus on the direct provision of services. This was to be complemented by 'care management', in which local social services departments were to take lead role. Care management was usually the responsibility of social workers who carried out an assessment of needs. The care plan was followed by the creation of a care plan. Social workers were given access to resources with which care was purchased. This was then monitored and altered to meet the changing needs of the individual. The main elements of the CPA are shown in Box 1.

Shepherd et al (1995) argued that the application of CPA to everyone in contact with specialist mental health services "makes targeting the most severely disabled extremely difficult". They pointed out that to reduce duplication, confusion and inefficient use of resources, the system should be rationalised into a single system combining the direct service elements of the CPA and the purchasing elements of care management. Marshall (1996) described the American concept of 'case management' as a combination of some aspects of care management and CPA. He felt that evaluation of care management had "provided little evidence of efficacy". The CPA, according to him, might have achieved some "approximation to assertive community treatment" with "proper funding and guidance". Instead, it had seen "lack of funding, mushrooming paperwork and extension to all psychiatric patients" without being fully evaluated. Easton \& Oyebode (1996) emphasised the logistical difficulties posed by CPA based on their experience of over 7000 patients. Tyrer et al (1995) gave evidence of increased hospitalisation of patients under care programming in a randomised

Box 1 Components of the Care Programme Approach

Systematic arrangements for assessing the health and social needs of people accepted into specialist mental health services

The formation of a care plan which identifies the health and social care required from a variety of providers

The appointment of a keyworker to keep in touch with the service user and monitor and coordinate care

Regular review and, where necessary, agreed changes to the care plan

Ashok Roy (Brooklands, Coleshill Road, Marston Green, Birmingham B37 7HL) is a consultant in learning disability psychiatry in North Warwickshire and North Birmingham Community Trusts. He is Medical Director of North Warwickshire Trust and a senior clinical lecturer in the Department of Psychiatry at Birmingham University. His interests include service development, access to primary care services, phenomenology of depression and ethical issues in learning disability, such as sterilisation and consent. 
control trial. Phelan (1996) suggested that this might indicate that CPA was helping services maintain contact with patients and highlighting unmet need. Increased hospitalisation might reflect increased patient contact with services and not necessarily mean a failure in the CPA. The publication of Building Bridges and The Spectrum of Care (Department of Health, 1995, 1996) saw the appearance of further guidance on improved inter-agency working and an audit pack for the CPA. White et al (1997), who provided additional guidance on eliciting risk, also highlighted risk management as an integral part of CPA.

In summary, although the CPA and care management were regarded as useful concepts, there were concerns expressed about the manner of their implementation.

\section{Modernising the CPA}

The publication of A First Class Service: Quality in the NHS (Department of Health, 1998a) heralded a more structured and coherent approach to quality of care. An element of this were "clear national standards for services and treatments, through National Service Frameworks". These were evidence-based standards of service provision, which would drive up quality in the NHS. This laid the foundation for the publication of A National Service Framework for Mental Health and Effective Care Co-ordination in Mental Health Services: Modernising the Care Programme Approach (Department of Health, 1999a,b). The latter document directs mental health and social services providers to:

- integrate the CPA with care management to form a single care coordination approach for adults of working age with mental health problems;

- appoint a lead officer to work across all agencies to deliver an integrated approach to Care Management and the CPA;

- apply CPA as a framework for mental health care to service users in residential settings and in the community and not use it simply as an after-care arrangement;

- introduce two levels of CPA: standard and enhanced;

- abolish supervision registers;

- change the name of the keyworker to 'care coordinator';

- reduce bureaucracy so that an undue burden is not placed on professionals whose prime responsibility is to provide clinical services;

- review and evaluate care plans at regular intervals and at the request of any member of the care team or the user or carer - at each meeting, the date of the next review must be set and recorded;

- collect data locally on service users and the numbers of people on the CPA;

- audit the quality of CPA implementation, care plans, attainment of treatment goals and the effectiveness of interagency working, including the views of service users;

- carry out risk assessment, early follow-ups of discharged patients and contingency plans for those on enhanced CPA;

- deliver services appropriate to the needs of service users through care planning;

- take account of the needs of carers and children and comply with the Carers Act 1995 and the National Service Framework standard on caring for carers; and

- take shared responsibility between the chief executive of the mental mealth provider trust and the partner director of social services to implement the changes.

It is anticipated that these changes will be officially implemented from April 2001. Local services can abolish supervision registers earlier if they have robust CPA arrangements in place.

The integration of CPA and care management should, therefore, lead to a seamless service having:

- a single point of referral;

- a unified health and social care assessment process;

- coordination of the respective roles and responsibilities of each agency; and

- access through a single process to the support and resources of both health and social care.

Features of an integrated system of the CPA and care management include: a single operational policy; joint training for health and social care staff; common risk assessment and management processes; shared information systems across health and social care; and agreement on the allocation of budgets.

The Mental Health National Service Framework sets a standard which requires that those on the CPA should be able to access services 24 hours a day, 365 days a year.

\section{Need for CPA in the field of learning disability}

There is strong evidence to suggest that mental health problems are common in people with learning disabilities and in most instances higher 
than corresponding figures in the general population. Corbett (1979) found a prevalence of schizophrenia of 3\% and an incidence of 3.5\%. Lund (1985) found similar prevalence in people with mild and moderate learning disability. Rates for affective disorder vary from 1.7\% (Lund, 1985) to 6.7\% (Moss et al, 1993), depending on the population studied and the screening and diagnostic tools used. The prevalence of personality disorders was found to be $25.4 \%$ in the Camberwell study (Corbett, 1979), 22\% in a hospital sample (Reid \& Ballinger, 1987) and 31\% in a community sample (Khan et al, 1997). The CPA is thus highly relevant for people with a learning disability, owing to the prevalence of mental health needs.

In an important national survey of learning disability services, Patton found that of 80 responses to a questionnaire regarding the use of CPA in learning disability services, only 29 were providing CPA (details available from the author upon request). Of this group, 24 included a risk assessment and 12 used a specialised system. A further 15 provided CPA for people with dual diagnosis using mental health databases, though in very small numbers. Thirty-six were not providing CPA. One of the reasons for this was that commissioners and local managers did not ask them to. Many were planning to use the CPA soon. The CPA has been seen in some services as relevant for the general population and not for people with a learning disability. Also, people with learning disability in contact with services do not necessarily have mental health needs and therefore the CPA is not relevant for them. She concluded that people in learning disability services needed more support to use CPA and incorporate good practice of care planning into the CPA process.

For people with a learning disability with mental health needs to derive maximum benefit from the CPA, professionals working in community teams, hospital-based teams and other settings must be in broad agreement with the criteria for eligibility for the CPA. Services must guard against the process becoming bureaucratic, so that it remains of benefit to clients. All people with a learning disability identified as having a mental health need must be assessed for need for the CPA. Some of the characteristics of people with a learning disability who are likely to be on an enhanced CPA are shown in Box 2 .

\section{Severe mental illness}

The working definition of severe mental illness, based upon the definition given in Building Bridges (Department of Health, 1995), is multi-dimensional
Box 2 Common characteristics of people with learning disabilities on an enhanced CPA

Being subject to Section 117 of the Mental Health Act (MHA) 1983

Being considered for discharge under Section 25 of the MHA 1983

Meeting the definition of severe mental illness

Needing a care plan with a high number of components, with input from several professionals (such as nursing, psychology, psychiatry and social work)

Having complex mental health needs with mental illnesses coexisting with neurological, developmental and personality disorders

Likely to or having history of harming themselves or being abused or neglected

Likely to or having history of harming property or other people

Showing offending behaviour

Showing poor cooperation with treatment and having poor insight

Having weak social and family support networks

and covers safety, informal and formal care, diagnosis, disability and duration (SIDDD).

- Safety: includes four components: unintentional self-harm (self-neglect), intentional selfharm, safety of others and abuse by others (physical, sexual, emotional or financial).

- Informal and formal care: includes help from informal carers, including friends and relatives, and from formal services, including day centres, paid staff, voluntary services, hospital admissions, medication and the Mental Health Act.

- Diagnosis: may include psychotic illness (including schizophrenia and affective disorder), dementia, severe neurotic illness, personality disorder and developmental disorder.

- Disability with impaired ability to function effectively in the community: including employment and recreation, personal care, domestic skills and interpersonal relationships.

- Duration of any of these for periods of 6 months and over.

They may display florid symptoms or suffer from a persistent enduring condition, and/or have suffered recurrent crises leading to frequent admission, and/or present significant risk to their own safety or that of others. 
This means that individuals with a primary diagnosis of learning disability without any additional mental health need will not meet the criteria for the CPA described above. People with multiple physical disability would benefit from care planning but would not be eligible for the CPA.

\section{Risk assessment}

Risk assessment and management are essential and ongoing parts of good mental health care for people with a learning disability. This is not a form-filling exercise but a dynamic process in which all service providers must participate by carrying out, sharing and recording assessed risk. Some of the common questions to be asked to obtain a risk profile in a person with a learning disability are shown in Box 3. The list is not exhaustive but forms a useful tool for screening for risk in a clinical setting.

A positive answer to any of these questions represents the presence of clinical risk. Other significant factors include history of poor compliance with treatment, recent discharge from hospital, having been detained under the Mental Health Act, suffering from certain types of personality disorder, especially those with prominent paranoid, emotionally unstable, impulsive and psychopathic traits. Risk factors are further exacerbated if the individual has poor social and family support networks. At the end of risk assessment, a judgement needs to be made about the complexity of the problems faced by the individual with a learning disability, the consequent complexity of the service needed and the risk to and from the individual.

Once the risk assessment is done and a decision is made to proceed with the CPA, care planning must be carried out.

\section{Care planning}

The first step in care planning is an assessment of clinical need through systematic evaluation. This then informs and drives the care plan. For people with learning disability, a full assessment must consist of five main domains of inquiry (Box 4).

\section{Psychiatric and behavioural problems}

The main psychoses, neuroses and personality disorders must be looked for, and predisposing (e.g.
Box 3 Risk assessment questions

Abuse by others?

Self-neglect?

Coexisting autistic traits?

Self-injury, overdose?

Suicidal ideation or threat?

Unacceptable social or sexual behaviour?

Fire setting?

Aggression or violence to staff, carer or others?

Alcohol or drug misuse?

Destruction of property?

Paranoid delusions or command hallucinations?

Severe mood abnormalities or swings? Coexisting personality disorder, hyperactivity?

family history), precipitating (e.g. bereavement) and maintaining factors (e.g. inadequate treatment) must be understood. This usually involves input from various professionals, such as psychiatrists, psychologists and community nurses. Special attention must be given to looking for longstanding problems commencing in childhood, for example, attention deficit hyperactivity disorder.

\section{Case example}

Richard was a 19-year-old man with moderate learning disability who had been showing inappropriate sexual familiarity towards female members of staff at his new college. This was attributed to lack of social skills. Only on closer assessment was it learnt that his behaviour had been associated with sleep disturbance and periods of preoccupation and withdrawal. His mother was found to have a chronic schizophrenic illness and she was undergoing treatment. Richard was diagnosed to be suffering from schizophrenia and within 3 months of starting treatment he had returned to college. He received regular out-patient supervision from the consultant and depot injections and support from the community nurse.

\section{Box 4 Domains for assessment of clinical need}

Psychiatric and behavioural

Developmental

Intellectual

Physical

Psychosocial 


\section{Developmental disorders}

Autism (pervasive developmental disorders) frequently coexists with learning disability. Berney (1997) quotes prevalence rates of severe learning disability in autism the range of $27-70 \%$. Scragg \& Shah (1994) found that the prevalence of Asperger's syndrome was greater in a special hospital than in the general population. It is important to look for autistic features in individuals, as they are often missed in adults. The characteristic range of disabilities in autism (including communication difficulties, ritualistic behaviour, poor empathy and a difficulty in generalising experience from one setting to another) make it difficult for people with the disorder to derive benefit from programmes not properly tailored to their needs.

\section{Case example}

Peter was a 31-year-old man with a mild learning disability who had a long history of getting into conflict where he worked, in a sheltered industrial therapy unit. Most recently he had assaulted a member of staff who had intervened when Peter threatened to attack another service user. He was remanded to a local psychiatric unit. On closer assessment, it was found that he fitted all the criteria for Asperger's syndrome. It was then noted that the staff had not known this and had not told him of any changes in the service early enough to prepare him for the change. He found this very anxiety-provoking. When the relevant changes were made and the staff received some training Peter was reintroduced to his job.

\section{Intellectual impairment (degree of learning disability)}

It is essential to determine if the level of learning disability is mild, moderate or severe. This can be clinically determined by measuring the range of ability an individual has on a variety of areas in such as self-care, communication, cognitive skills and social skills. This helps to plan the appropriate level of long-term support and assistance individuals may need. Communication therapists, occupational therapists and psychologists may be called upon to provide further help with assessment and subsequent care planning. People with mild learning disability may find themselves falling between the learning disability and general adult services. In keeping with spirit of the CPA, their complex mental health needs should be managed jointly between the generic mental health and learning disability services. Some individuals with borderline learning disability and schizophrenia may show a cognitive decline during their illness.

Communication assessment can reveal a range of difficulties in both expression and comprehension.
Specific problems need to be identified if mistakes are to be avoided in giving information. Semantic and pragmatic disorders are being increasingly identified in people who appear to be able to hold a conversation but who take in only a fragment of what is said to them. The frustrations caused by these difficulties can lead to into behavioural problems.

\section{Physical disorders}

It is important to diagnose and plan care and treatment for associated physical conditions which may be of aetiological significance, such as Down's syndrome, or contribute to the existing disabilities, such as cerebral palsy or sensory disabilities, or which may be coincidental, such as obesity. The management of these conditions forms a vital part of the final care plan.

Epilepsy is commonly associated with learning disability and mental health problems. The complex relationships between these disabilities needs to be understood in each case for a successful care plan to be drawn up. Diabetes and thyroid disease can also exacerbate mental and behavioural problems. Existing behavioural phenotypes need to be identified, as they are often associated with certain behaviours, such as self-injury in Cornelia de Lange syndrome or hyperphagia and dysphoria in Prader Willi syndrome.

An evaluation must be made to detect abnormalities in vision and hearing, as these can affect the efficacy of treatment.

The use of the CPA for the management of physical comorbidity has been proposed recently by Santhouse \& Holloway (1999). They advocate physical review and screening, as well as close liaison with general practitioners.

\section{Psychosocial situations}

These encompass a variety of situations, such as problems related to employment, housing and economic circumstances, social environment, childhood adversity and upbringing, family circumstances and lifestyle. Difficulties in caring can result from inability of the primary support group to provide good physical care or a positive emotional atmosphere conducive to optimal development. Other problems include imminent breakdown of residential placement and possible homelessness, adverse life events such as bereavement, or loss of social and leisure opportunities.

An assessment of an individual with learning disability and mental health needs must be systematic in covering all these areas. Problems identified in each area should result in identification of corresponding service need, leading to a better 
care plan. The characteristics of a good care plan are shown in Box 5.

The principles of effective care planning are illustrated by the following case example.

\section{Case example}

Lily is 42-year-old woman with Down's syndrome who lives with her elderly parents. She attends a day centre and goes there by coach. In the last two months, she had been aggressive to other clients both on the coach and at the centre, which led to her being excluded. Her social worker referred her to the community learning disability team. The community nurse found the parents exhausted trying to contain Lily's aggression and through discussion with her general practitioner (GP) initiated a referral to the learning disability psychiatrist.

Lily was admitted for in-patient assessment, where it was found that she was low in mood. She was waking up early, non-communicable, frequently irritable and had little interest in her favourite activities such as using the computer. She had to be encouraged to look after her personal needs. She was diagnosed with a moderately severe depressive illness and hypothyroidism in addition to a severe learning disability.

Her care plan therefore covered the following areas:

- treatment of depression with appropriate antidepressant (medical staff);

- treatment for hypothyroidism (medical staff);

- assessment of communication skills (speech therapist);

- assessment of daily living skills to plan appropriate community support (occupational therapist);

- discussion of future placement with family members and Lily (social worker);

- continued observations and monitoring of progress on the ward (nursing staff); and

- arranging weekly multi-disciplinary reviews (consultant psychiatrist).

Box 5 Characteristics of a good care plan

Identify the interventions and anticipated outcomes

Record all the actions necessary to achieve the agreed goals

In the event of disagreement, include the reasons

Give an estimated time-scale for achieving or reviewing outcomes or goals

Detail the contributions of all the agencies involved

Include appropriate crisis and contingency plans
At a multi-disciplinary review meeting attended by medical staff, nurses, communication therapists, occupational therapists, social worker, Lily and her parents, it was felt that Lily had responded well to treatment with antidepressants and thyroxine and was ready to make a fresh start. It was also agreed that she would find it more stimulating and enjoyable living in a staffed group home with friends, visiting her parents regularly. Staff from the day centre were happy for Lily to resume her attendance there once Lily was discharged from hospital.

Based on the assessment, the social worker found a suitable group home, which Lily visited and liked. After a trial period, Lily moved on a permanent basis. The social worker who had a central role in planning Lily's life agreed to take on the responsibility of care coordinator.

\section{Role of care coordinator}

Once the decision is made to place an individual on the CPA, a care coordinator is appointed. The care coordinator should be best placed to oversee care planning and resource allocation. In people on standard CPA, where only one professional is involved, that person should be the care coordinator. In reality, the role of care coordinators has the potential to be of greatest benefit for clients on enhanced CPA. Here coordinators need to be in close contact with the person with a learning disability, so that changes in circumstances can be communicated to other members of the care team. Care plans and crisis plans can then be suitably updated. This means that health and social service managers must support these individuals in fulfilling their role. For the attributes of a care coordinator see Box 6 .

\section{Training issues}

For the CPA to be successfully used for people with a learning disability, it is vital that there is joint working between health and social agencies in both the learning disability and generic mental health services. This can be successfully achieved if there is joint training for all the professionals involved. This is particularly relevant for nurses, psychologists, psychiatrists and social workers. It is not sufficient to have individuals trained in learning disabilities working alongside of those trained in mental health. All professionals need to have a good understanding of the nature and psychopathology of mental health problems in the general population. They need to have, in addition, 
a shared understanding of: the specific clinical presentations of mental health problems in people with a learning disability at different levels of functioning; the potential diagnostic difficulties encountered; coexistence of multiple disabilities and problems; and the multiplicity of management strategies available. This shared view, combined with the widest possible access to a range of services, is essential for delivering relevant, high quality services to people with a learning disability and mental health needs. A lack of this dual perspective makes it is easy wrongly to attribute signs and symptoms of a mental illness or autism to the learning disability itself. Without training and perspective it is highly unlikely that care coordination will be integrated. Strong links to the criminal justice system should also be established.

The responsibility for ongoing training lies with health and social services through the CPA lead officer working across agencies.

\section{Crisis and contingency planning}

People with a learning disability on enhanced the CPA require as part of their care plan crisis and contingency plans. These are aimed at preventing crises by detailing arrangements to be used where the care coordinator is unavailable and the care plan cannot be provided. It must give the contact numbers of interim care staff who can avert major difficulties by timely intervention. Community-based rapid response teams may be able to give urgently needed carers support to cope with a person with a learning disability, thus avoiding admission to hospital.

\section{Setting, context and supports}

The CPA is not simply a framework for after-care of hospitalised patients. In modern learning disability services, the majority of people with mental health needs are supported and treated in community settings, often in their own home. Planning must give primacy to the assessed needs of the clients and their carers with, wherever possible, support from an advocate. The client's general practitioner, along with other members of the primary care team such as practice nurses, should play a key role in maintaining continuity of care.

Information needs to be shared across health, social services and the criminal justice systems. It
Box 6 Attributes of a care coordinator

Understanding of mental health needs in people with a learning disability

Knowledge of the client and his or her family as well as awareness of issues such as race, culture and gender

Knowledge of community services and role of relevant agencies and teams

Organisational skills

Access to resources

must be remembered that to make CPA effective it must be provided in a coordinated manner wherever the individual lives. This can happen only if relevant information is available to whoever is providing the service. Respect for confidentiality needs to be balanced against issues of safety and public interest. This can prove challenging when planning and providing services for people with a learning disability who have committed offences and who have mental health problems. Information systems must, in addition to providing accurate and up-todate details of clients, help with audit and future service planning. While integrated information systems may take time to develop, local interagency agreements are the key to improving the quality of services.

\section{Conclusion}

The publication of A National Service Framework for Mental Health (Department of Health, 1999a) and Effective Care Co-ordination in Mental Health Services: Modernising the Care Programme Approach (Department of Health , 1999b) signify an important opportunity to set high standards for services for people with a learning disability having additional mental health needs. Signposts for Success (Department of Health, 1998b) has set out good practice guidelines on most aspects of these services.

It is now imperative for primary care groups and trusts, learning disability services, health authorities and social services along with the criminal justice system to use the new CPA to provide more coordinated services based on both assessed clinical need and safety. The improved frameworks now available should reduce duplication and increase the provision of seamless services with the clients and their carers at its centre. 


\section{References}

Berney, T. P. (1997) Autism and Asperger's Syndrome. In Psychiatry in Learning Disability (ed S. G. Read), pp. 150-184. London: W. B. Saunders.

Corbett, J. (1979) Psychiatric morbidity and mental retardation. In Psychiatric Illness and Mental Handicap (eds P. Snaith \& F. E. James), pp. 11-25. Ashford: Headley Brothers.

Department of Health (1990) NHS and Community Care Act. London: HMSO.

- (1990) Caring for People. London: DoH.

- (1995) Building Bridges. A guide to Arrangements for InterAgency Working for Care and Protection of Severely Mentally Il People. London: $\mathrm{DoH}$.

- (1996) The Spectrum of Care. London: DoH.

- (1998a) A First Class Service: Quality in the New NHS. London: $\mathrm{DoH}$

- (1998b) Signposts for Success in Commissioning and Providing Health Services for People with Learning Disabilities. London: DoH.

- (1999a) A National Service Framework for Mental Health. London: DoH.

- (1999b) Effective Care Co-ordination in Mental Health Services: Modernising the Care Programme Approach. London: DoH.

Easton, C. \& Oyebode, F. (1996) Administrative demands of care programme approach. British Medical Journal, 312, 1540.

Khan, A., Cowan, C. \& Roy, A. (1997) Personality disorders in people with a learning disability: a community survey. Journal of Intellectual Disability Research, 41, 324-330.

Lund, J. (1985) The prevalence of psychiatric morbidity in mentally retarded adults. Acta Psychiatrica Scandinavica, $72,563-570$

Marshall, M. (1996) Case management: a dubious practice. British Medical Journal, 312, 523-524.

Moss, S., Patel, P., Prosser, H., et al (1993) Psychiatric morbidity in older people with moderate and severe learning disability. I: Development and reliability of the patient interview (PASADD). British Journal of Psychiatry, 163, 471-480.

Phelan, M. (1996) Care management. British Medical Journal, 312, 1539-1540.

Reid, A. H. \& Ballinger, B. R. (1987) Personality disorder in mental handicap. Psychological Medicine, 20, 983-987.

Santhouse, A. \& Holloway, F. (1999) Physical health of patients in continuing care. Advances in Psychiatric Treatment, 5, 455-462.

Scragg, P. \& Shah, A (1994) Prevalence of Asperger's syndrome in a secure hospital. British Journal of Psychiatry, 165, 679-682

Shepherd, D., King, C., Tilbury, J., et al (1995) Implementing the Care Programme Approach. Journal of Mental Health, 4, 261-274.

Tyrer, P., Morgan, J., van Horn, E., et al (1995) A randomised controlled study of close monitoring of vulnerable psychiatric patients. Lancet, 345, 756-759.

White, K., Roy, D. \& Hamilton, I. (1997) ABC of mental health: community mental health services. British Medical Journal, 314, 1817.

\section{Multiple choice questions}

1 The CPA is:

a meant solely for patients discharged from psychiatric hospitals

$\mathrm{b}$ aimed at health and social services

c applicable to all age groups

$\mathrm{d}$ a new concept being launched for the first time with the Mental Health National Service Framework

e not applicable in prisons.
2 Modernising the CPA involves:

a appointing care coordinators

b giving consultant psychiatrists devolved budgets to develop services

C using supervision registers to monitor people with severe mental illness

$d$ collating information from the use of the CPA and audit services jointly

e involving GPs and carers more in treatment decisions.

3 Applying the CPA to people with a learning disability and additional mental health needs: a involves the psychiatrist in all cases

$\mathrm{b}$ is best done after a multi-professional assessment

c is more likely to lead to increased hospitalisation

$\mathrm{d}$ may lead to greater joint working between learning disability and generic mental health services

e will inevitably lead to increased bureaucracy.

4 Care planning for people with a learning disability:

a usually follows an initial risk assessment

b is solely focused on the psychiatric diagnosis

c can take into account the quality of family support

$\mathrm{d}$ is best done in a specialised setting, preferably in a hospital

e is followed by a series of pre-planned review meetings to check on progress with treatment.

5 The following are likely to reduce risk for people with learning disability on enhanced CPA

a having a care coordinator with knowledge of local services

b having regular visits to their GP

c reducing community support after 1 month if there have been no problems

d having a crisis plan filed with their medical records at the psychiatric hospital where they were treated 1 year ago

e suspecting a relapse of a mental illness when they stop eating and going to their day service.

\section{MCQ answers}

\begin{tabular}{|c|c|c|c|c|}
\hline 1 & 2 & 3 & 4 & 5 \\
\hline a $F$ & a $\mathrm{T}$ & a $F$ & a $\mathrm{T}$ & a $\mathrm{T}$ \\
\hline b $T$ & b F & b $T$ & b F & b $\mathrm{T}$ \\
\hline c $F$ & c $F$ & c $F$ & c $\mathrm{T}$ & c $F$ \\
\hline d F & d $T$ & d $T$ & d F & d F \\
\hline $\mathbf{F}$ & e $T$ & e $F$ & e $T$ & e $T$ \\
\hline
\end{tabular}

